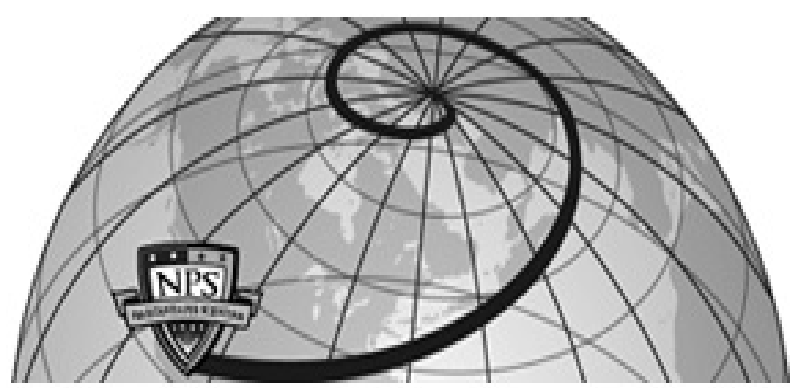

Calhoun: The NPS Institutional Archive DSpace Repository

\title{
Network Structure and Innovation Ambiguity Effects on Diffusion in Dynamic Organizational Fields
}

Gibbons, Deborah E.

Academy of Management Journal, 2004, Vol. 47, No. 6, 938951.

https://hdl.handle.net/10945/46065

This publication is a work of the U.S. Government as defined in Title 17, United States Code, Section 101. Copyright protection is not available for this work in the United States.

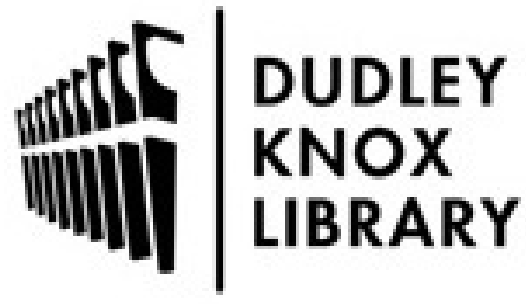

http://www.nps.edu/library
Calhoun is the Naval Postgraduate School's public access digital repository for research materials and institutional publications created by the NPS community. Calhoun is named for Professor of Mathematics Guy K. Calhoun, NPS's first appointed -- and published -- scholarly author.

Dudley Knox Library / Naval Postgraduate School 411 Dyer Road / 1 University Circle Monterey, California USA 93943 


\title{
NETWORK STRUCTURE AND INNOVATION AMBIGUITY EFFECTS ON DIFFUSION IN DYNAMIC ORGANIZATIONAL FIELDS
}

\author{
DEBORAH E. GIBBONS \\ Naval Postgraduate School
}

\begin{abstract}
Computational modeling simulated innovation diffusion through six prototypical interregional network structures and two distributions of partnering tendencies in dynamic organizational fields. Compared to regional constraints, connections among all geographic regions decreased clearly beneficial innovation diffusion (a low-threshold adoption model) but increased ambiguous innovation diffusion (a social influence model). Compared with uniform partnering tendencies, normally distributed partnering tendencies increased diffusion of ambiguous innovations. Overall, local and interregional network structures interacted with the observability of an innovation's benefits to determine diffusion.
\end{abstract}

As an organizational population develops, networks of relations form among its members. Adaptive change can diffuse through these networks (Kraatz, 1998), but structural disparities may determine whether an innovation sweeps through the field or languishes in obscurity. To the extent that innovation is necessary for the population to thrive, interorganizational structures that support diffusion processes are crucial. By exploring structural effects on innovation transfer at the system level, the research presented in this article provides guidance for network-building interventions and intelligent design of growing networks.

Given the difficulty of measuring networks at an industry level, it is not feasible to systematically compare structural effects on global diffusion using empirical data. Further, published studies focus primarily on situations in which an innovation has successfully diffused, so scholars have limited information about conditions that repress diffusion. Finally, empirical observations do not allow tests of alternative conditions that could change outcomes. A practical solution is to simulate nonlinear dynamics using parameters drawn from empirical work, as has been increasingly recommended by social and organizational scientists (e.g., Anderson, 1999; Kamps \& Masuch, 1997; Repenning, 2002).

Through computational modeling, this research replicated naturally occurring aspects of interorganizational networks and revealed their influences on diffusion in growing populations. Concurrent implementation of population ecology and net-

Thanks to Christopher Gibbons and C. J. Lee for help with the simulation code, model validation, and data management. Thanks to Wenpin Tsai and to three anonymous reviewers whose input significantly improved this article. working principles created a virtual world. Within that world, virtual experiments tested how distributions of partnering tendencies, centralities of initial adopters' contacts, and interregional network structures influenced the likelihood, speed, breadth, and level of diffusion.

The likelihood that diffusion will occur reveals a network's suitability for innovation transfer. The speed with which an innovation diffuses then affects the level of payback to early adopters because faster diffusion extends the benefits more quickly to others. Network impacts on diffusion speed are equally relevant for early movers who want to deter diffusion and for policy makers who would rather speed innovation through a field. Policy makers and strategists also need to recognize network effects on the breadth of diffusion among regions because geographic dispersion may cause regional discrepancies in technological or conceptual progress. Finally, increased understanding of structural influences on diffusion level can facilitate development of more efficient interorganizational networks.

\section{THEORETICAL BACKGROUND}

Organizational fields, which have been defined as populations of organizations that occupy the same or interrelated environmental niches (DiMaggio \& Powell, 1983), change over time. For this study, I narrowly defined an organizational field as including organizations that produce related outputs, use related resources, and rely on similar technologies. Every organizational field develops unique networks shaped by regional constraints and organizational partnering tendencies. Structural characteristics of each network then affect its capability to diffuse various kinds of innovations. 
Because of the organizational and technological similarities among the members of a field, an innovation that benefits one member can also benefit the others.

\section{Regional Constraint on Networks}

Geographic proximity of organizations increases opportunities for interaction, so regional network clusters arise naturally. External threats, shared culture and ethics, similar interests, and preexisting familiarity with the other organizations further encourage collaboration within a region (Doz, Olk, \& Ring, 2000). The same influences can be expected to yield more ties to neighboring regions than to distant ones. All else being equal, most interactions will occur within regions, some interaction will occur with neighboring regions, and minimal interaction will occur with distant regions.

In most industries, regional attributes moderate the proximity effect. Ability to obtain knowledge (Sidorenko \& Findlay, 2001) and innovate (Ritsila, 1999) varies across regions, and regions that include a wider range of capabilities, experiences, and resources are better able to incorporate external knowledge (Strambach, 2002). They become central in global structures, while less innovative regions remain peripheral. For example, organizations based in the dominant economic clusters of Europe, North America, and East Asia tend to partner with others from those three clusters. Within each cluster, leading countries may act as gatekeepers for information and resource flows to neighboring areas, as Japan has done in East Asia (Little, 1999). Even patterns of Internet presence (Sternberg \& Krymalowski, 2002) and of business-to-business e-commerce (Kshetri \& Dholakia, 2002) reveal strong regional limitations.

Patterns of regionalization vary. For example, in agriculture the interaction of organizations tends to be influenced by proximity, but in the pharmaceutical industry interregional hierarchies constrain interaction among organizations (Porter \& Stern, 2001). Although each network is unique, distinct patterns can be represented with prototypes such as unconstrained, decentralized, chain, hierarchical, and cliquish interregional structures. These interregional (macro) structures can be expected to influence the diffusion of innovations through a field. Figure 1 graphically depicts six prototypical patterns of regional networking.

\section{Organizational Partnering Tendencies}

The amount of variance in the centrality of organizations within a network differs from network to network (Newman, Strogatz, \& Watts, 2001). Some organizational fields provide equal opportunities for members to collaborate, while others feature variance in members' ability to develop partnerships. These distinct patterns of partnering tendencies create distinct network structures.

Many researchers have argued that network centralities follow a Poisson distribution, which results from uniform partnering tendencies, yet empirical research has demonstrated variety in centrality distributions (Barabasi \& Albert, 1999). Stable differences in partnering tendencies, reflecting organizations' unique opportunities and actions, have been observed (Gulati, 1995). Normal distribution of partnering tendencies is particularly likely to occur as some organizations fall below and others exceed the mean. Whether uniform or normally distributed, the pattern of partnering tendencies is a defining characteristic of a network, yet researchers know little about its effect on the diffusion of innovation.

\section{Network Structures and Diffusion}

Interorganizational ties can disseminate innovation (Davis, 1991) and foster adaptation (Kraatz, 1998), but structural aspects of networks regulate this diffusion process (Gulati, 1998). In sparsely networked populations, such as the residents of the United States (Milgram, 1967) or the members of interlocked corporate boards (Robins \& Alexander, 2004), a few bridging ties connect local social clusters. Although some researchers have reported that increasing ties among clusters facilitates diffusion, others have found the opposite.

Simulated "small-world" models (see Watts [1999b] for an overview) have tested effects of network constraint on the spread of disease and knowledge. Increasing ties between groups, holding within-group density constant, increases the likelihood of an epidemic (Moore \& Newman, 2000). In contrast, maximal knowledge distribution occurs when 95 percent of ties are local, and only 5 percent are distant (Cowan \& Jonard, 1999). These discrepant results imply that the diffusion effect of interregional structures depends on what is transferring.

Diffusion of a clearly beneficial innovation rests on potential adopters' access to knowledge. When a potential adopter observes innovation benefits, expected risk decreases and the likelihood of adoption increases (Wejnert, 2002). In this situation, interorganizational ties serve as information channels, and thresholds for adoption are low. Cowan and Jonard's results suggest that regional barriers facilitate the diffusion of clearly beneficial innova- 
FIGURE 1

Interregional Network Structures ${ }^{\mathrm{a}}$

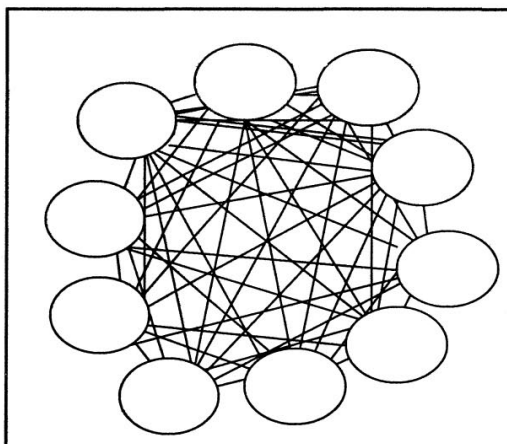

Structure 1: Unconstrained network

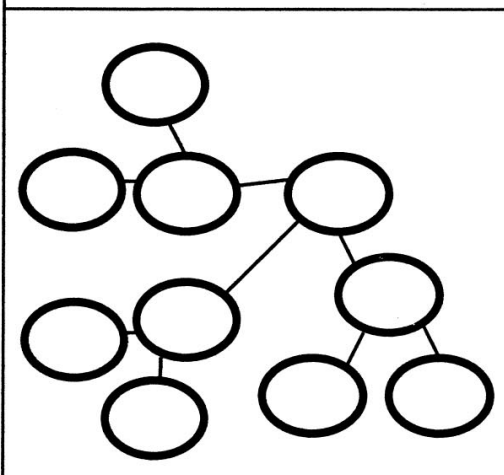

Structure 4: Hierarchy among regions

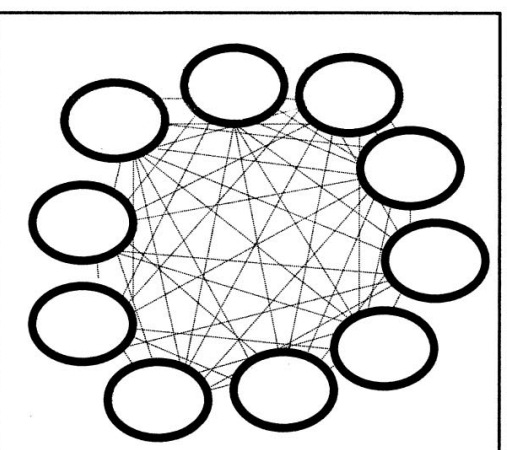

Structure 2: Decentralized interregional structure

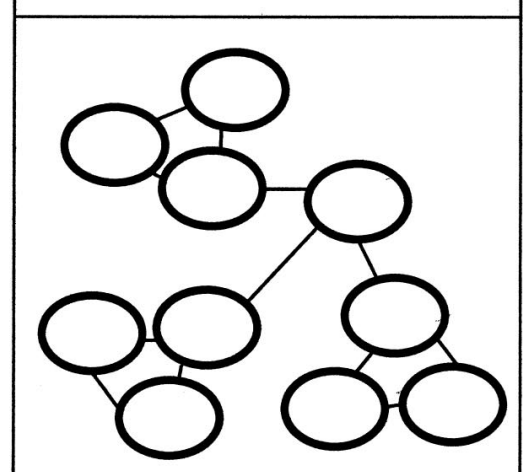

Structure 5: Central region connecting cliques via one region per clique

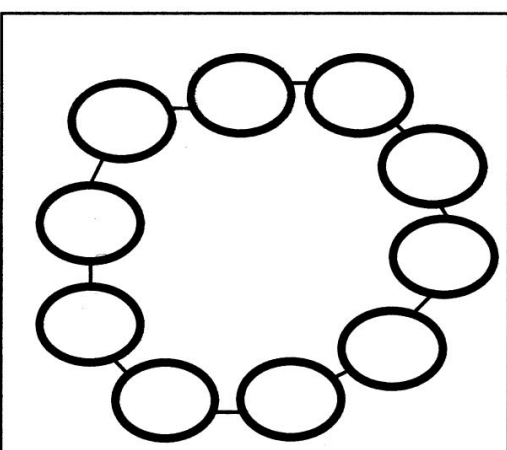

Structure 3: Regions connected in a chain

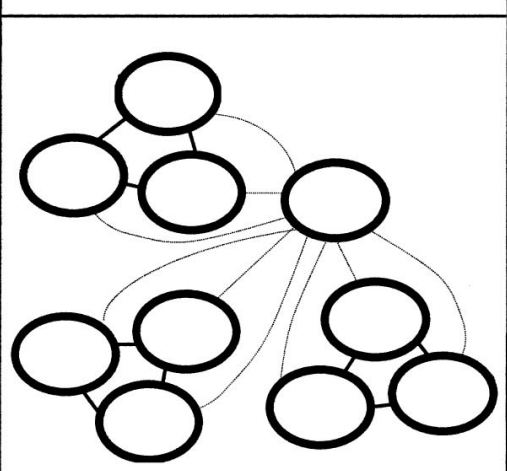

Structure 6: Central region connecting cliques via scant ties to all regions

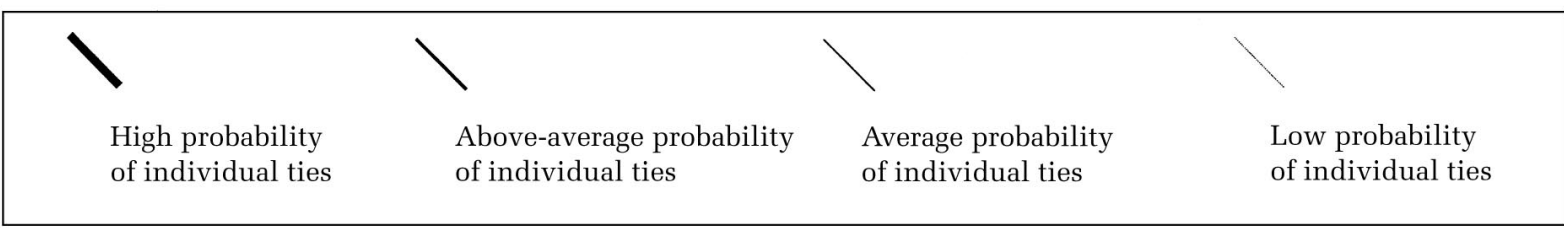

${ }^{a}$ Average partnering tendency is held approximately constant at .10.

tions. Specific interregional constraints are likely to have varying influences on diffusion.

When information about an innovation is ambiguous, subjective information from contacts plays a major role in the decision to adopt (DiMaggio \& Powell, 1983). When objective information is absent, the likelihood of adoption increases with the proportion of adopters among one's contacts. This is a contagion process, like the process that drives an epidemic, so greater connection among regions can be expected to facilitate diffusion of ambiguous innovations. Specific interregional structures are also likely to yield varying effects on this process.
Hypothesis 1. At a system level, interregional network structures have different impacts on the diffusion of clearly beneficial innovations and the diffusion of ambiguous innovations.

Within a macro structure, distributions of partnering tendencies affect organizational networking and the overall structure of the network. Many leaders in the public sector pursue uniform distribution of partnering tendencies among the organizations in their regions. Industrial associations likewise create formal structures that are designed to provide uniform partnering opportunities to all 
potential members. These efforts generally rest on an assumption that uniform partnering tendencies maximize benefits for everyone in the system and, by extension, for the system as a whole. While this assumption may be true for some kinds of activities, it is unlikely to hold true for all diffusion processes.

Variance in partnering tendencies occurs naturally, creating a range of organizational centralities, constraints, and opportunities within a social network. This range may produce a population in which some organizations are heavily influenced by a small number of innovating contacts because they have few competing relationships. Early conversion of the less central organizations could then contribute to the overall proportion of adopters and move the system toward a critical mass for widespread diffusion. Because an innovation's ambiguity affects its adoption process, variance in partnering tendencies may differently influence clearly beneficial and ambiguous innovation diffusion.

Hypothesis 2. Normal distribution of interorganizational partnering tendencies fosters greater diffusion of innovations than does uniform distribution of partnering tendencies. The extent of this effect depends on innovation ambiguity.

At an organization level, centralities of initial adopters establish the breadth of opportunity for the diffusion of all innovation types (Valente, 1996). Although they have received minimal research attention, the centralities of first adopters' contacts also play a role in all types of diffusion. Low centrality makes these organizations susceptible to first adopters' influence because they receive little contrary information. This information void increases the likelihood of initial diffusion from first adopters to their contacts, but very low centrality among the contacts may stifle diffusion because these organizations are thus less able to serve as conduits to the rest of the system. High centrality could also undermine diffusion, despite the increased dissemination potential, because central organizations may overlook initial adopters among their plethora of contacts. These competing processes imply a positive relation with systemwide diffusion to the point at which signals from initial adopters are overwhelmed by the volume of information from nonadopting partners.

Hypothesis 3. Increasing centrality among initial adopters' contacts positively influences diffusion only to the point at which communication with nonadopters overshadows inputs from initial adopters.
In summary, interregional structures, distributions of partnering tendencies, and the centralities of initial adopters' contacts influence the likelihood, pattern, and extent of diffusion. The following section maps theoretical concepts of population growth, network development, and diffusion onto corresponding simulation representations and experimental procedures.

\section{MODELING DIFFUSION THROUGH DYNAMIC POPULATIONS}

\section{Modeling Approach}

Individual actions can accumulate to create unexpected systemwide effects (Schelling, 1969) that are best understood through computational modeling (Robins, Pattison, \& Woolcock, 2002). In this study, the environment for the simulated diffusion process is a dynamic population of organizations that develops under empirical parameters. The innovations fall into two categories: those having clearly observable benefits and those whose equivalent benefits are not observable. Each innovation diffuses through every network structure from the same starting point in each of 100 growing organizational fields.

In this study's simulation, the principles governing organization births and deaths are based on U.S. government statistics and existing work in population dynamics (Amburgey, Kelly, \& Barnett, 1993; Lomi \& Larsen, 1996). The diffusion processes are grounded in social networks research (e.g., Granovetter \& Soong, 1983; Valente, 1995) and institutional theory (DiMaggio \& Powell, 1983). Table 1 summarizes the theoretical constructs tested here and their representations in the model.

\section{Organizational Populations and Networks}

Legitimacy, which results from proximity to similar organizations, increases organizational foundings and survival rates (Hannan \& Carroll, 1992). When an area becomes crowded, competition depresses foundings and increases the probability of failure (Lomi \& Larsen, 2001), but established and adapted organizations are more likely to survive (Baum, 1996). More than a third of American start-up companies fail in their first three years (Office of Advocacy, 2001), but the risk of failure decreases over time (U.S. Small Business Administration, 1998). A newly changed organization also experiences increased risk because any innovation can disrupt organizational processes. If the organization weathers the change, risk declines, as envi- 
TABLE 1

Theoretical Foundations for Model Parameters

\begin{tabular}{|c|c|c|}
\hline Parameter & Theoretical Construct & Representation in Model \\
\hline \multicolumn{3}{|l|}{ Population } \\
\hline Births & Likely under sparse population. & Occurs given three neighbors. \\
\hline \multicolumn{3}{|l|}{ Deaths } \\
\hline Due to isolation & Likely if few or no similar organizations. & Occurs given 0 or 1 neighbor. \\
\hline Due to overcrowding & $\begin{array}{l}\text { Likely if too many organizations compete for } \\
\text { same resources, mitigated by stability and fit. }\end{array}$ & Probabilistic given $7-8$ neighbors. \\
\hline Newness/stability & & $p$ reduced by age/10 $(\leq .3)$. \\
\hline Environmental fit & & $p$ reduced by adoption (.1). \\
\hline \multicolumn{3}{|l|}{ Network } \\
\hline $\begin{array}{l}\text { Regional structures } \\
\text { Unconstrained } \\
\text { Decentralized } \\
\text { Regional chain } \\
\text { Hierarchy } \\
\text { Central region, } \\
\text { regional cliques } \\
\text { (2) }\end{array}$ & $\begin{array}{l}\text { Test effects of prototypical interregional } \\
\text { network structures on systemwide diffusion } \\
\text { (see “Structures,” Figure 1). }\end{array}$ & $\begin{array}{l}\text { Pairwise tie probabilities weighted within versus across } \\
\text { regions (retaining overall density). }\end{array}$ \\
\hline $\begin{array}{l}\text { Partnering tendencies } \\
\text { Similar } \\
\text { Varying }\end{array}$ & $\begin{array}{l}\text { Represent fields where organizations: } \\
\text { Have similar tendency to tie } \\
\text { Vary in tendency to tie }\end{array}$ & $\begin{array}{l}\text { Partnering tendency distribution: } \\
\text { uniform }(p=.1) \\
\text { normal (mean } p=.1 \text {, sd. }=.03 \text { ) }\end{array}$ \\
\hline \multicolumn{3}{|l|}{ Innovation } \\
\hline Benefit observability & Represent innovation aspects: & Two adoption models: \\
\hline Clearly positive & Clearly observable benefits & $\begin{array}{l}\text { Low-threshold adoption occurs if } \geq .15 \text { of an } \\
\text { organization's ties have adopted innovation }\end{array}$ \\
\hline Ambiguous & Difficult-to-assess benefits & $\begin{array}{l}\text { Social influence ( } p=\text { percentage of organization's } \\
\text { ties that have adopted innovation) }\end{array}$ \\
\hline
\end{tabular}

ronmentally appropriate adaptation improves chances for survival (Amburgey, Kelly, \& Barnett, 1993).

Together, legitimacy, competition, adaptation, and stability regulate the distribution of organizations in a field. Ongoing development of the field, in turn, affects innovation diffusion in two ways. Congenital learning and selection processes directly impact the proportion of adapted organizations within the field. Regional boundaries and availability of potential partners indirectly impact patterns of diffusion by constraining network formation.

Each organizational field in the simulation is represented by 400 locations in a square $(20 \times 20)$ lattice. The lattice is divided into ten (20 rows by 2 columns) regional blocks. As in the real world, the eastern and western regions adjoin. Each organization's neighborhood includes the 8 (5 each on the northern and southern boundaries) locations adjoining it.

The population component of the simulation is a variation of Conway's game, Life (Gardner, 1970).
Three principles are applied in each time period to every location. First, three organizations adjoining an unoccupied location result in a birth. This event indicates resource availability in a geographic region that is not saturated with competitors. Second, given no neighbor or one neighbor, geographic isolation leads to death. This event reflects the illegitimacy ascribed to unusual or unfamiliar businesses in a local community. Third, if seven or eight organizations adjoin an occupied location, competition for resources may lead to death. Probability parameters (Table 1) reflect decreasing failure rates during the first three years of operation, an adoption benefit, and short-term risk due to adaptation shock (Hannan \& Freeman, 1984).

Within each population, network formation is influenced by locally specified constraints on random generation of ties. In a stable structure, organizational centralities remain similar over time (Doreian, 1986), so the simulation retains each organization's tendency to create ties as long as that organization exists. 


\section{Experimental Design}

Conditions in the virtual experiment conducted here included uniform and normally distributed partnering tendencies, six interregional structures, and two innovation types. Measured variables included region of initial adoption, number of initial adopters that immediately failed, regional and overall population densities, centralities of initial adopters, and centralities of initial adopters' contacts.

In the initial population and for subsequent births, each organization was assigned a value between 0 and 1 to represent its partnering tendency (Table 1). A low density was chosen to reflect the real-world network inclination to have sparse contacts relative to the number of potential contacts (Watts, 1999a). In one experimental "condition," all organizations were equally likely $(p=.1)$ to create ties. In the other, the tendency to create ties was drawn from a normal distribution (mean $p=.1$, s.d. $=.03$ ) with a range from 0 to 1 . Every pair's baseline probability of creating a reciprocal tie (a pairwise tie probability) equaled the average of their partnering tendencies. When organizations failed, their ties were destroyed. Organizations that subsequently appeared in those locations drew partnering tendencies from the appropriate distribution, and the network updated itself probabilistically.

The simulation creates macro structures (Figure 1 and Table 1) by scaling tie probabilities within and among regions. Unconstrained structure 1 links organizations in a worldwide network. All other structures scale within-block tie probabilities by 4 , preclude ties between some regions, and scale other tie probabilities to create network prototypes. Decentralized structure 2 scales between-region tie probabilities by .67. Regional chain structure 3 scales adjacent-region tie probabilities by 3 . Hierarchical structure 4 scales tie probabilities between connected regions by 3.33. Structure 5 represents regional cliques that are connected through a central region. The between-region tie probabilities are scaled by 2.5. Structure 6 duplicates the configuration in structure 5, spreading the central region's external tie probabilities across all other regions.

The simulation uses two adoption models to represent the diffusion of clearly beneficial innovations and the diffusion of ambiguous innovations. The clear-benefits model applies a 15 percent adoption threshold to all organizations. Under the ambiguous-benefits model, each organization considers adoption when any of its contacts adopts, with the probability of adoption being equal to the percentage of adopters among the contacts. If the organization fails to adopt, it does not reconsider unless another contact adopts.

\section{Simulation Procedure}

Each cycle of the simulation begins when one seeds an empty field with organizations at a density of approximately .15 and then allows the simulation parameters to structure the population. The probabilistic Game of Life runs for 15 time periods, and the population matrix is stored. Pretests showed densities ranging from 0 to .66 after 15 periods.

For each condition, ties develop among existing organizations, and five initial adopters are placed in one region. This is the only point in the simulation at which new organizations arise in locations without exactly three neighbors. If no organization exists at a selected location, a new organization is created and incorporated into the network. The population is updated, new ties form, and new adoptions are recorded. During the population development phase, a new organization includes the innovation at birth if the majority of the organization's geographical neighbors have adopted.

The simulation loops 25 times in every population for each of 48 conditions (two partnering tendency distributions, six macro structures, two innovation types, and two locations for initial adopters). Each sequence begins with an identical population and configuration of initial adopters. When all 48 conditions have run, one cycle is completed. Parameters are reset, a new population develops, and the process repeats for 100 cycles.

\section{Analyses}

I used logistic regression analysis to determine which factors increased the likelihood of diffusion. Ordinary least squares (OLS) analyses were used to test effects on diffusion level, speed, and breadth. Diffusion level was defined as the percentage of total population that eventually adopted. Diffusion speed was defined as the average number of new adopters per time period prior to stability. Time of stability was defined (1) as the last period in which an organization was born, died, or adopted an innovation, (2) as the last period before a pattern began to repeat, or (3) in a few cases where the system did not verifiably reach stability, as the 25th time period. Diffusion breadth was defined as the number of regions containing adopters at stability.

Predictors included the parameterized variables and the average centrality of organizations tied to initial adopters. I calculated the average centralities of initial adopters' contacts using only data from initial adopters that survived at least one time period. 
TABLE 2

Diffusion Outcome Cell Means ${ }^{\mathrm{a}}$

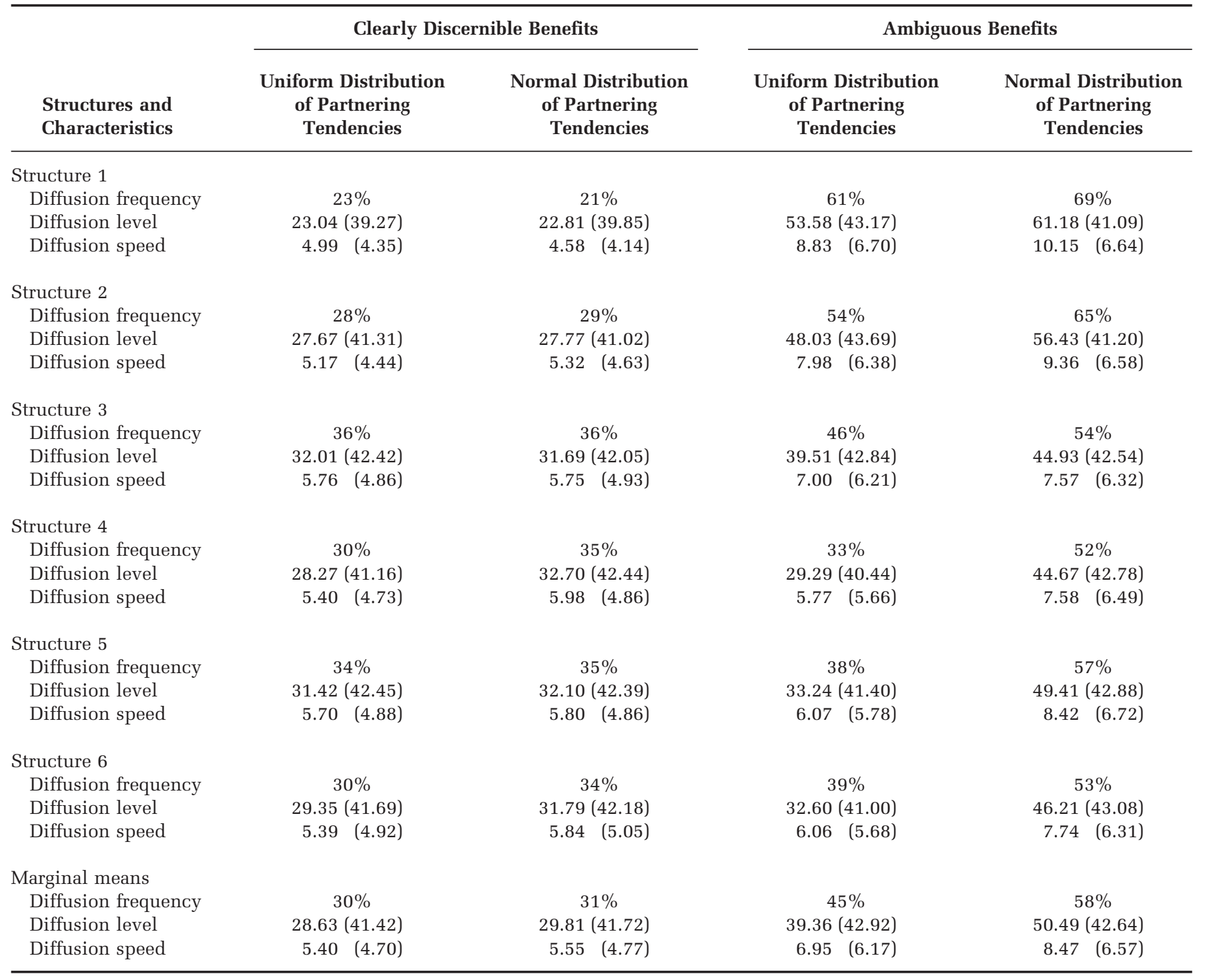

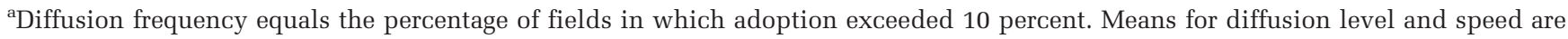
reported with standard deviations in parentheses; $n$ ranges from 195 to 198 per cell.

\section{RESULTS}

Examination of outcomes indicated a bimodal distribution of diffusion levels. Given that five adopters were seeded into each field, I defined a diffusion subset as all cases in which at least six organizations adopted the innovation. Excluding fields containing five or fewer adopters at stability yielded 2,852 cases. Of the 1,948 cases that did not evidence any diffusion, 83 involved death of all five initial adopters without transmission to other organizations. These cases were excluded from analysis, leaving 4,717 data points in the full sample and 2,852 data points in the diffusion subset. Initial plots indicated that network centralities had curvilinear effects on diffusion level, so I included higher-order terms in regression equations.

For the logistic regression analysis, fields in which more than 10 percent of the population adopted were classified as in the diffusion category. Fields in which 10 percent or fewer adopted were classified as in the nondiffusion category. Because of the bimodal distribution, very few cases fell between 10 and 60 percent diffused. The model correctly categorized 81.4 percent of nondiffusion cases and 61.7 percent of diffusion cases.

OLS regression models predicting diffusion level, speed, and breadth included controls for population density, the location and centrality of 
FIGURE 2

Diffusion over Time through Unconstrained, Decentralized, Chain, and Hierarchical Interregional Structures

Clearly Beneficial Innovation: Low-Threshold Diffusion Model

(2a) Full Sample

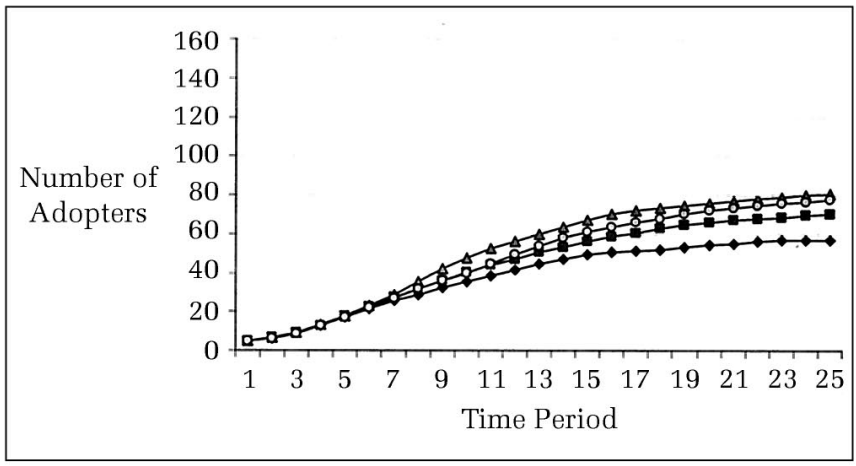

(2b) Diffusion Subset

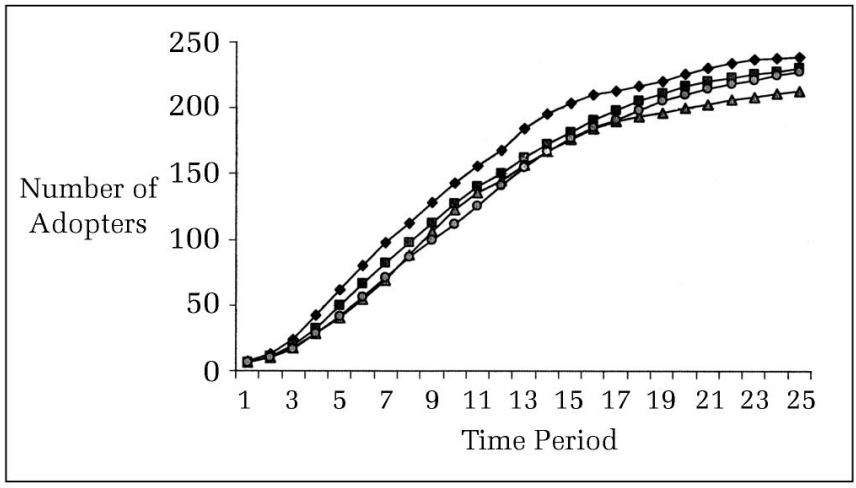

Ambiguous Innovation: Social Influence Diffusion Model
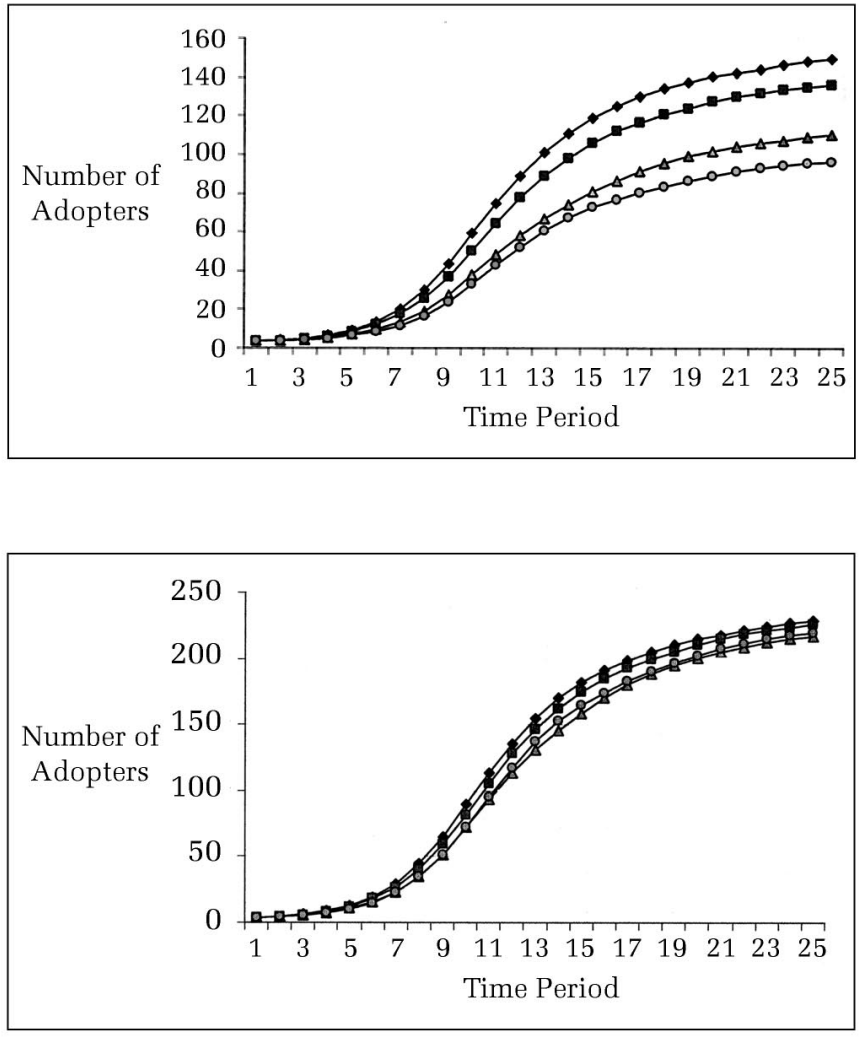

$\multimap$ Structure 1 $\rightarrow-$ Structure 2 $\checkmark-$ Structure 3 - - Structure 4

initial adopters, and dead initial adopters. I transformed diffusion level using the natural logarithm before running regressions.

Table 2 reports diffusion frequency (the proportion of cases in which adoption exceeded 10 percent), mean diffusion level, and mean diffusion speed by structural condition for each type of innovation.

Interregional network structures differently impacted diffusion of clearly beneficial and ambiguous innovations at the system level. Structures 1 and 2 increased the probability, level, speed, and breadth of ambiguous innovation diffusion, while structure 1 decreased the probability and level of clearly beneficial innovation diffusion.

Effects of macro structures on the two types of innovation diffusion become apparent through ex- amination of cell means in Table 2. For visibly beneficial innovations, diffusion frequency was highest through the interregional chain (structure $3)$. Both the chain and the hierarchical clique structure (5) increased the level and speed of clearly beneficial innovation diffusion, while frequency, level, and speed were lowest through structure 1. In contrast, decentralized structures 1 and 2 exceeded the population mean for the frequency, level, and speed of ambiguous innovation diffusion.

Cumulative adoption curves for both innovation types appear in Figure 2. For visual clarity, the graphs include only structures 1 through 4 . The clearly beneficial innovations produce a logarithmic curve under all structures, a curve that is commonly observed in diffusions based on product attributes (see Mahajan, Muller, \& Wind, 2000). 
FIGURE 3

Effects of Uniform versus Normally Distributed Partnering Tendencies on Diffusion Levels and Speeds ${ }^{\mathrm{a}}$

Clearly Beneficial Innovation: Low-Threshold Diffusion Model

(3a) All Diffusion Levels

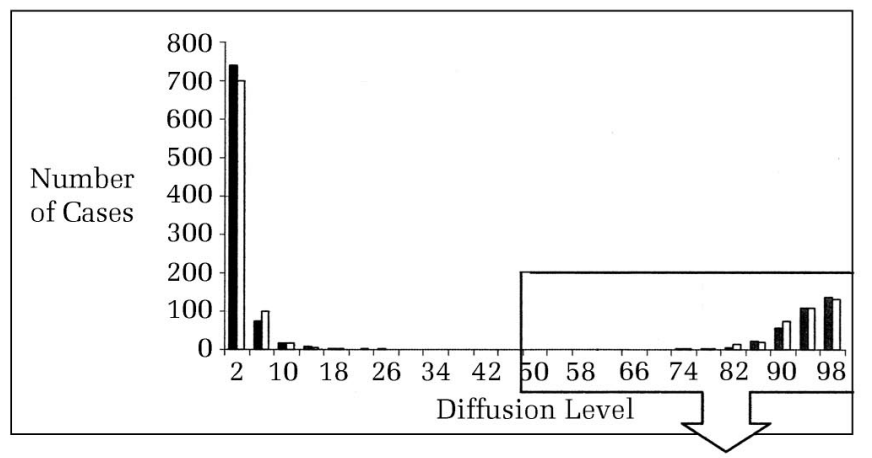

(3b) Successful Diffusion

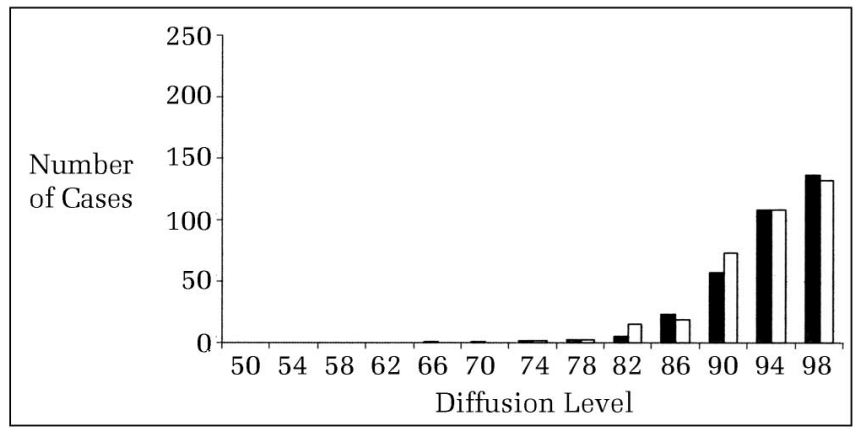

(3c) Diffusion Speeds

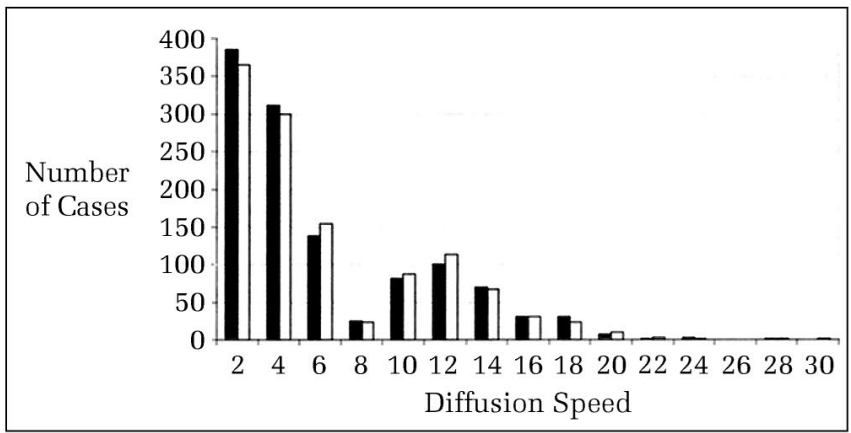

Ambiguous Innovation: Social Influence Diffusion Model
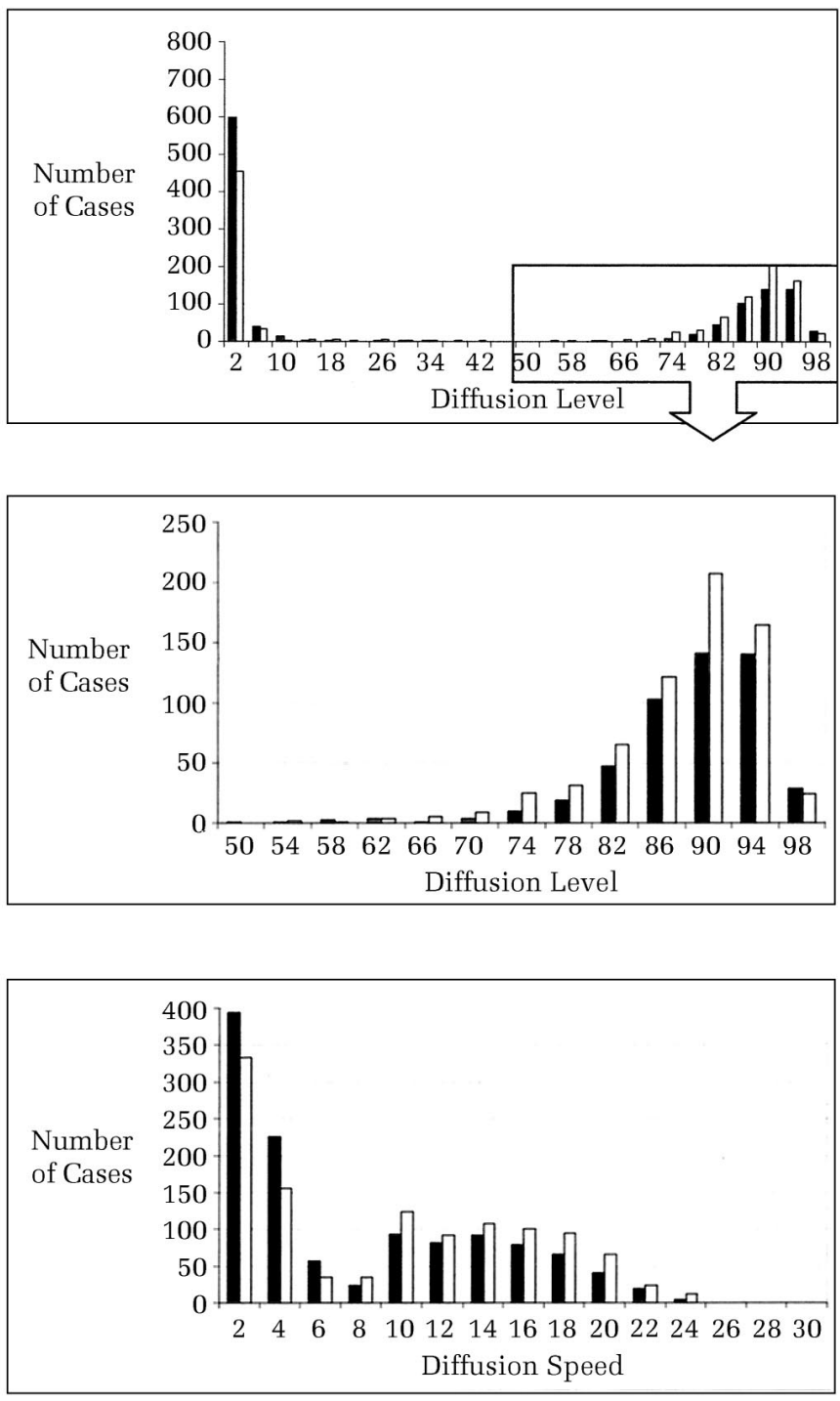

= Uniform partnering tendency $\quad \square=$ Normally distributed partnering tendency

${ }^{a}$ Diffusion level is the percentage of a population that had adopted at stability. Diffusion speed is the average number of adopters per time period prior to stability.

Ambiguous innovations produce a familiar S-curve, a form typical of social influence processes. In the full sample, structures 3 and 4 outperform structures 1 and 2 for clearly beneficial innovations, and structures 1 and 2 outperform structures 3 and 4 for ambiguous innovations. In the diffusion subset, the less constrained structures 1 and 2 support greater diffusion of both innovations. This pattern indicates that interregional con- straint facilitates initial diffusion of a clearly beneficial innovation, but broader communication across regions hastens diffusion that does occur.

Normal distribution of partnering tendencies fostered greater diffusion of ambiguous innovations than did uniform partnering tendencies, increasing diffusion frequency from 45 percent to 58 percent (Table 2). Regression results indicate that normally distributed partnering tendencies also increased 
diffusion level, speed, and breadth for ambiguous innovations. Distribution of partnering tendencies had little effect on clearly beneficial innovations. Significant interaction between distributions of partnering tendencies and innovation type can be seen graphically in Figure 3. Figure 3a shows the full range of diffusion levels for each type of innovation, broken out by distribution of partnering tendencies. Figure $3 \mathrm{~b}$ enlarges the graph for cases in which an innovation diffused. This limited range is more likely to parallel empirical studies because most failed diffusions do not come to researchers' attention. Figure 3c reveals the pattern of partnering distribution effects on diffusion speed for each type of innovation.

Increasing centralities of initial adopters' contacts had an inverted-U-shaped relation with diffusion, peaking at 1.33 standard deviations below the mean for likelihood of diffusion and at 1.13 standard deviations above the mean for diffusion level in the full sample. In the diffusion subset, higher contact centrality increased diffusion level only at the bottom of the range. Higher contact centrality consistently increased diffusion speed and breadth. These results indicate that high contact centrality reduces the chance that an innovation will diffuse, but it speeds and broadens any diffusion that occurs.

\section{DISCUSSION AND CONCLUSION}

This article began with the claim that simulation of diffusion processes in dynamic organizational fields can provide insights unavailable through other approaches. By creating a virtual world that operates under empirically based rules of population development and network constraint, the current simulation enabled examination of situated effects of network attributes on fieldwide diffusion. Interregional structures, distributions of partnering tendencies, and centralities of initial adopters' contacts played complex roles in the diffusion of innovation. Further, the large proportion of nondiffusion cases indicates that a similarly large number of failed diffusion processes occurs in the real world. Consideration of the full range of outcomes through simulation reveals joint effects of interregional structures and partnering tendencies on diffusion failure and on successful diffusion processes.

Figure 4 illustrates population growth and diffusion in a typical field through structure 1, in which ties are unconstrained. Each set of small graphs depicts population development and diffusion under one condition at four-period intervals. The top row in each pair shows population development. The second row in each pair shows which existing organizations had adopted at that time. All three examples begin with the same population and ini- tial adopters. By comparing the first and second examples, one can see different processes evoked by clearly beneficial and ambiguous innovation diffusion under normal distributions of partnering tendencies. The third example depicts development of the field given ambiguous benefits and uniform partnering tendencies. Comparison with the second example reveals less and slower diffusion given uniform partnering tendencies than given normal distribution of partnering tendencies. Overall results indicate that a decentralized interregional network with diverse organizational tendencies to create ties is optimal for fostering the diffusion of ambiguous innovations. This network configuration is the least effective for diffusion of clearly beneficial innovations.

Regional constraints on network formation improve the likelihood that clearly beneficial innovations will diffuse. Macro structures that form a chain (structure 3) and moderately connected cliques (structure 5) are particularly conducive to launching the process. Although diffusing innovations travel more slowly through constrained networks than through decentralized structures, the higher success rate indicates that interregional clustering fosters diffusion of clearly beneficial innovations. This finding aligns with prior work on knowledge exchange processes indicating that high levels of regionalization enhanced diffusion (Cowan \& Jonard, 1999) and extends that work by distinguishing among patterns of interregional constraint. Although effects of specific interregional structures depend on innovation type, hierarchy does not facilitate diffusion of either innovation.

Local aspects of the networks also influenced systemwide diffusion. At low levels, increasing centrality among initial adopters' contacts increased diffusion. By the midrange, increasing contact centrality began to decrease adoption behavior, even as it accelerated and broadened successful diffusions. The role of first adopters' contacts as gateways between initial adopters and the rest of the network created a need for moderate centrality in order to maximize diffusion.

\section{Model Limitations and Future Research}

The prototypical network structures represented in the simulation differ in their alignment with naturally occurring networks. Because regional barriers are influential and nearly ubiquitous, they affect any field that extends beyond local boundaries. The unconstrained network is less realistic than the others because natural networks exhibit more clustering than occurs through random generation of ties (Albert \& Barabasi, 2002). The struc- 


\section{FIGURE 4}

Population Growth and Diffusion through a Decentralized Network

(4a) Low-Threshold Model, Normally Distributed Partnering Tendency
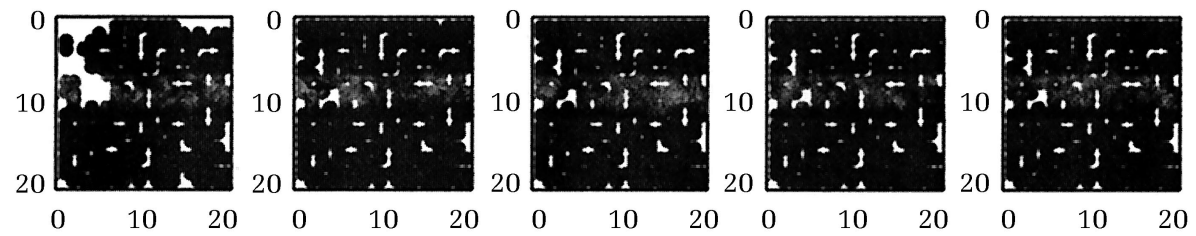

Population $=246$ Population $=267$ Population $=268$ Population $=268$ Population $=268$
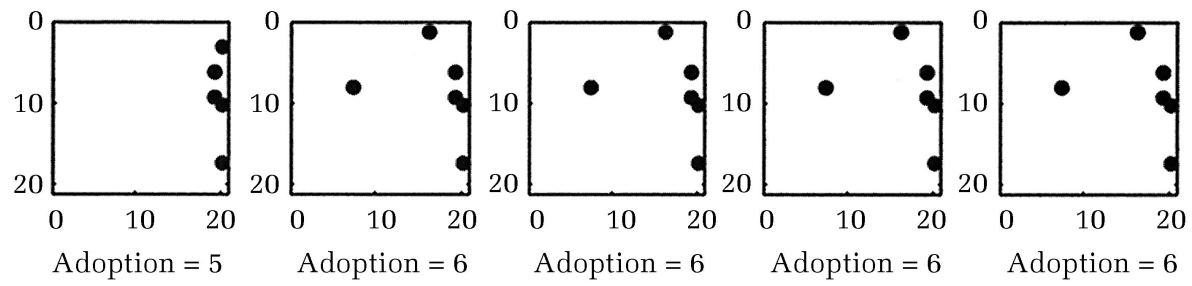

(4b) Social Influence Model, Normally Distributed Partnering Tendency
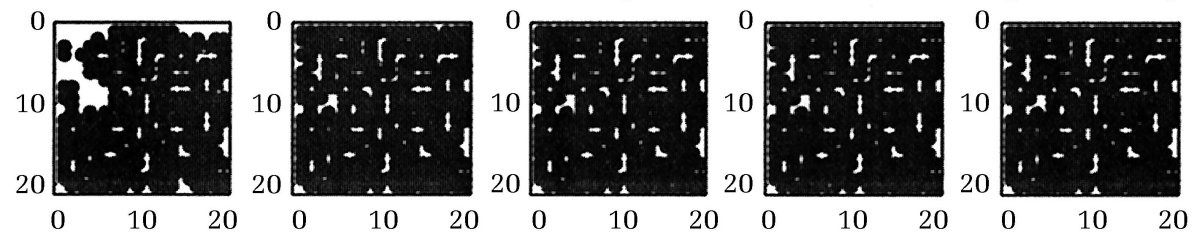

Population $=246$ Population $=268$ Population $=267$ Population $=267$ Population $=267$
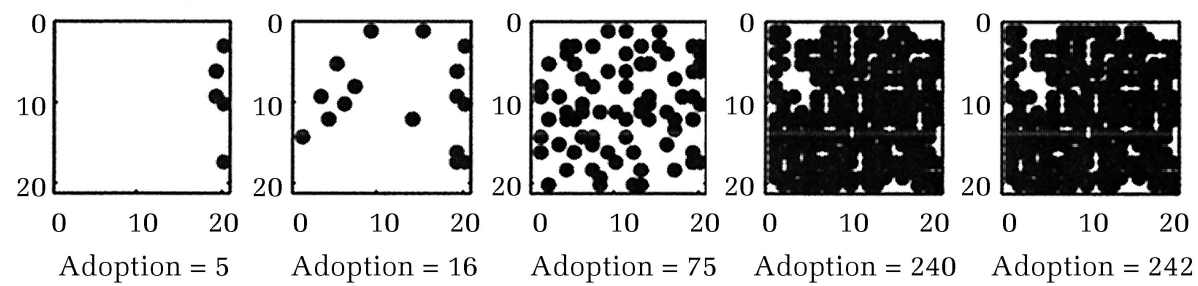

(4c) Social Influence Model, Uniform Partnering Tendency
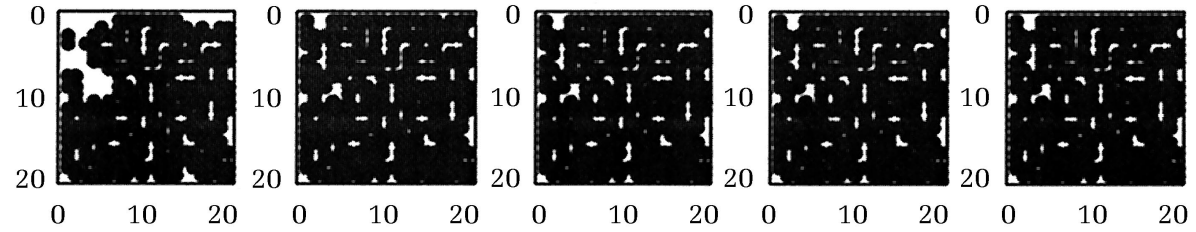

Population $=246$ Population $=266$ Population $=265$ Population $=265$ Population $=265$
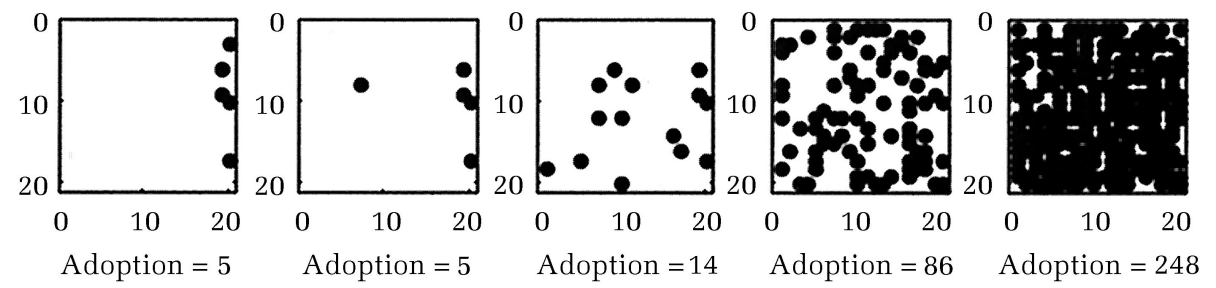

ture of this model probably underestimates clique formation. Along similar lines, uniform partnering tendencies are less likely to occur than normally distributed tendencies. Preexisting ties influence creation of future ties (Gulati, 1995), and new organizations are more likely to link with an existing organization that is central than with one that is peripheral (Barabasi \& Albert, 1999). 
Variation in network density within and across growing organizational fields is an interesting area for future research. Although higher network density increases flows of information or of disease, density may have more complex effects on innovation adoption. In particular, increasing density without increasing number of initial adopters might hinder diffusion if the initial adopters become less salient to their contacts. This situation would produce an inverted-U-shaped relationship between density and diffusion, similar to the relation between centrality among first adopters' contacts and diffusion. The models presented here do not include individual or environmental factors that lead an organization to actively seek or suppress information regarding innovation. Levels of competition may systematically influence these behaviors and moderate structural effects on patterns of diffusion. Further empirical tests of informationsharing behaviors across organizations will be necessary before such effects can be confidently simulated.

Another interesting direction for ongoing research would be to examine the effect of diffusion on organizational centrality. Holding constant the tendency to create ties and making no assumptions about nonstructural characteristics, one might expect early adopters to become more central over time. Increasing centrality of early adopters has been observed among individuals (Burkhardt \& Brass, 1990), but it has not been examined as a purely structural outcome among organizations. If an innovation increases likelihood of survival, members of initial adopters' networks that also adopt are more likely than nonadopters to survive. Given a constant partnering tendency, those whose existing contacts survive will accumulate new relationships with minimal loss of old ones. Extending this effect across an adapting population, earliest adopters would increase their ties and their gatekeeping ability relative to the rest of the field. These interactions can be tested through future simulation models that integrate principles of population ecology, network development, and innovation diffusion.

Population development and diffusion are both nonlinear processes subject to chaotic effects. Despite relatively low levels of randomness in the simulation compared to the real world, chaos played a large role in population-level change. Estimates of population and structural effects on diffusion level indicated that 69.3 percent of the variance resulted from divergence following initial adoption. This surprisingly high random effect indicates that much of the variance observed among organizational fields reflects small, idiosyncratic events that occur during population and network development. It further implies that tiny deviations in current structures may blossom into vast differences within a few years. This possibility should encourage large institutions, such as governments and industry associations, that would like to increase the innovation capacity of organizational networks. Small, strategic nudges can have immense effects.

\section{Network Design Applications}

The key to successful network design rests in knowing where and how to nudge the system. From this simulation, I draw three practical conclusions. First, interregional structures that maximize diffusion of ambiguous innovations may deter diffusion of clearly beneficial innovations. One needs to structure for whichever kind of innovation is most important or most prevalent in a field. Strategic bridge building between unconnected regions can improve the transfer of ambiguous innovations. Allowing sparsely connected regional clusters can improve the transfer of clearly beneficial innovations.

Second, interregional hierarchy does not facilitate innovation diffusion. Regardless of innovation type, hierarchical structures among regions work against innovation diffusion apart from economic, cultural, and local factors. Because established social structures tend to reinforce themselves, barriers between regions may resist change. Rather than battle hierarchical macro structures, change agents might directly introduce innovations to peripheral regions and let them percolate through the system. Interactions around the innovations could then create new ties (Burkhardt \& Brass, 1990) that gradually restructure the network.

Finally, the distribution of ties has cumulative effects on systemwide diffusion. An ideal local design for either type of innovation includes highly central innovating organizations and moderately central contacts. Because variance in partnering tendencies can increase the diffusion potential of a network, efforts to equalize network access may be counterproductive.

\section{Conclusion}

By modeling diffusion through growing networks in dynamic populations, this study has established foundations for new computational and empirical research, as well as guidelines for management and public policy. Comparison of structural effects on adoption of clearly beneficial versus ambiguous innovations introduces a practical approach to understanding distinct diffusion processes. Beyond 
simple contagion, specific diffusion consequences of prototypical interregional structures, distributions of partnering tendencies, and centralities of initial adopters' contacts are identified here. Because the same structure that enables diffusion of one innovation can squelch diffusion of another, understanding these interactions is fundamental for individuals and organizations that want to build effective networks.

\section{REFERENCES}

Albert, R., \& Barabasi A. L. 2002. Statistical mechanics of complex networks. Reviews of Modern Physics, 74: $47-97$.

Amburgey, T. L., Kelly, D., \& Barnett, W. P. 1993. Resetting the clock: The dynamics of organizational change and failure. Administrative Science Quarterly, 38: 51-73.

Anderson, P. 1999. Complexity theory and organization science. Organization Science, 10: 216-232.

Barabasi, A. L., \& Albert, R. 1999. Emergence of scaling in random networks. Science, 286: 509-513.

Baum, J. A. C. 1996. Organizational ecology. In S. R. Clegg, C. Hardy, \& W. R. Nord (Eds.), Handbook of organization studies: 78-114. London: Sage.

Burkhardt, M. E., \& Brass, D. J. 1990. Changing patterns or patterns of change: The effect of change in technology on social structure and power. Administrative Science Quarterly, 35: 104-127.

Cowan, R., \& Jonard, N. 1999. Network structure and the diffusion of knowledge. Working paper MER 99028, MERIT, University of Maastricht.

Davis, G. F. 1991. Agents without principles? The spread of the poison pill through the intercorporate network. Administrative Science Quarterly, 36: 583613

DiMaggio, P. J., \& Powell, W. W. 1983. The iron cage revisited: Institutional isomorphism and collective rationality in organizational fields. American Sociological Review, 48: 147-160.

Doreian, P. 1986. On the evolution of group and network structure II: Structures within structure. Social Networks, 8: 33-64.

Doz, Y. L., Olk, P. M., \& Ring, P. S. 2000. Formation processes of R\&D consortia: Which path to take? Where does it lead? Strategic Management Journal, 21: 239-266.

Gardner, M. 1970. The fantastic combinations of John Conway's new solitaire game "life." Scientific American, 223: 120-123.

Granovetter, M., \& Soong, R. 1983. Threshold models of diffusion and collective behavior. Journal of Mathematical Sociology, 9: 165-179.

Gulati, R. 1995. Social structure and alliance formation patterns: A longitudinal analysis. Administrative Science Quarterly, 40: 619-652.

Gulati, R. 1998. Alliances and networks. Strategic Management Journal, 19: 293-317.

Hannan, M. T., \& Carroll, G. R. 1992. Dynamics of organizational populations: Density, legitimation, and competition. New York: Oxford.

Hannan, M. T.,\& Freeman, J. H. 1984. Structural inertia and organizational change. American Sociological Review, 49: 149-164.

Kamps, J., \& Masuch, M. 1997. Partial deductive closure: Logical simulation and management science. Management Science, 43: 1229-1245.

Kraatz, M. S. 1998. Learning by association? Interorganizational networks and adaptation to environmental change. Academy of Management Journal, 41: 621643.

Kshetri, N., \& Dholakia, N. 2002. Determinants of the global diffusion of b2b e-commerce. Electronic Markets, 12(2): 120-129.

Little, S. 1999. Global production and global consumption: Designing organizations and networks for the next century. Creativity and Innovation Management, 8(1): 8-19.

Lomi, A., \& Larsen, E. R. 1996. Interacting locally and evolving globally: A computational approach to the dynamics of organizational populations. Academy of Management Journal, 39: 1287-1321.

Lomi, A., \& Larsen, E. R. 2001. Failure as a structural concept: A computational perspective on age dependence in organizational mortality rates. In A. Lomi \& E. R. Larsen (Eds.), Dynamics of organizations: Computational modeling and organizational theories: 269-303. Menlo Park, CA: AAAI Press/MIT Press.

Mahajan, V., Muller, E., \& Wind, Y. 2000. New-product diffusion models. Boston: Kluwer Academic.

Milgram, S. 1967. The small world problem. Psychology Today, 1(1): 60-67.

Moore, C., \& Newman, M. E. J. 2000. Epidemics and percolation in small-world networks. Physical Review E, 61: 5678-5682.

Newman, M. E. J., Strogatz, S. H., \& Watts, D. J. 2001. Random graphs with arbitrary degree distributions and their applications. Physical Review E, 64: 1-19.

Office of Advocacy. 2001. Minorities in business, 2001. http://www.sba.gov/advo/stats/min01.pdf. Retrieved September 18, 2003.

Porter, M., \& Stern, S. 2001. Innovation: Location matters. Sloan Management Review, 42(4): 28-36.

Repenning, N. P. 2002. A simulation-based approach to understanding the dynamics of innovation implementation. Organization Science, 13: 109-127.

Ritsila, J. J. 1999. Regional differences in environments 
for enterprises. Entrepreneurship \& Regional Development, 11: 187-202.

Robins, G., \& Alexander, M. 2004. Small worlds among interlocking directors: Network structure and distance in bipartite graphs. Computational \& Mathematical Organization Theory, 10(1): 69-94.

Robins, G., Pattison, P., \& Woolcock, J. 2002. Small and other worlds: Global network structures from local processes. Working paper, University of Melbourne.

Schelling, T. 1969. Models of segregation. American Economic Review, Papers and Proceedings, 59: $488-493$.

Sidorenko, A., \& Findlay, C. 2001. The digital divide in East Asia. Asian-Pacific Economic Literature, 15(2): $18-30$.

Sternberg, R., \& Krymalowski, M. 2002. Internet domains and the innovativeness of cities/regions-Evidence from Germany and Munich. European Planning Studies, 10: 251-273.

Strambach, S. 2002. Change in the innovation process: New knowledge production and competitive citiesThe case of Stuttgart. European Planning Studies, 10: $215-231$.

U.S. Small Business Administration. 1998. The state of small business: A report of the president, 1997 (Office of Advocacy). Washington, DC: United States Government Printing Office.
Valente, T. W. 1995. Network models of the diffusion of innovations. Cresskill, NJ: Hampton Press.

Valente, T. W. 1996. Social network thresholds in the diffusion of innovations. Social Networks, 18: 6989.

Watts, D. J. 1999a. Small worlds: The dynamics of networks between order and randomness. Princeton, NJ: Princeton University Press.

Watts, D. J. 1999b. Networks, dynamics, and the smallworld phenomenon. American Journal of Sociology, 105: 493-527.

Wejnert, B. 2002. Integrating models of diffusion of innovations: A conceptual framework. In J. Hagan \& K. S. Cook (Eds.), Annual review of sociology, vol. 28: 297-326. Palo Alto, CA: Annual Reviews.

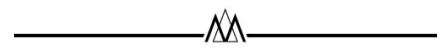

Deborah E. Gibbons (degibbon@nps.edu) is an assistant professor at the Graduate School of Business and Public Policy in the Naval Postgraduate School. She received her Ph.D. in organizational behavior and theory from Carnegie Mellon University. Her current research involves assessment, benchmarking, and development of interorganizational networks, particularly as they relate to collaboration, diffusion, and community building in the public sector.

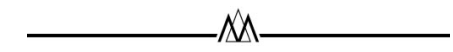


Copyright of Academy of Management Journal is the property of Academy of Management and its content may not be copied or emailed to multiple sites or posted to a listserv without the copyright holder's express written permission. However, users may print, download, or email articles for individual use. 